\title{
Effect of the stellar absorption line centre-to-limb variation on exoplanet transmission spectrum observations
}

\author{
F. Yan $^{1}$, E. Pallé ${ }^{2,3}$, R. A. E. Fosbury ${ }^{4}$, M. G. Petr-Gotzens ${ }^{4}$, and Th. Henning ${ }^{1}$ \\ 1 Max Planck Institute for Astronomy, Königstuhl 17, 69117 Heidelberg, Germany \\ e-mail: fyan@mpia.de \\ 2 Instituto de Astrofísica de Canarias, C/ vía Láctea, s/n, 38205 La Laguna, Tenerife, Spain \\ 3 Dpto. de Astrofísica, Universidad de La Laguna, 38206 La Laguna, Tenerife, Spain \\ ${ }^{4}$ European Southern Observatory, Karl-Schwarzschild-Str. 2, 85748 Garching bei München, Germany
}

Received 26 November 2016 / Accepted 17 March 2017

\begin{abstract}
Transit spectroscopy is one of the most commonly used techniques for exoplanet atmosphere characterisation. This technique has been used to detect ionised and neutral species in exoplanet atmospheres by comparing the observed stellar lines in and out of transit. The centre-to-limb variation (CLV) of the stellar lines across the stellar disk is an important effect for transmission spectroscopy, since it results in a change of stellar line depth when the planet transits different parts of the stellar disk. We reanalysed the transit data of HD 189733b taken with the HARPS spectrograph to study the CLV effect during transit. The transmission light curve of the Na I D line so obtained shows a clear imprint of the CLV effect. We used a one-dimensional non-LTE stellar spectral model to simulate the CLV effect. After applying the correction, the measurement of the Na I absorption in the atmosphere of HD 189733b becomes better determined. We compared the CLV effect of HD 189733b to that of HD 209458b. The CLV effects are different for these two benchmark planetary systems and this is attributed to their different stellar effective temperatures and transit impact parameters. We then explored the general CLV effect that occurs during exoplanet transits. Normally, a star with a lower effective temperature exhibits a stronger CLV effect and its CLV feature extends over a relatively broad wavelength range. The transit impact parameter (b) describes the transit trajectory on the stellar disk and thus determines the actual manifestation of the CLV effect. We introduced a b-diagram which describes the behaviour of the CLV effect as the function of different impact parameters. With improving observational precision, a careful modelling and correction of the CLV effect is necessary for exoplanet atmosphere characterisation using transit spectroscopy.
\end{abstract}

Key words. planets and satellites: atmospheres - techniques: spectroscopic - stars: atmospheres

\section{Introduction}

Atmospheric characterisation is a rapidly expanding branch of exoplanet research. One of the most commonly used methods for this is transit spectroscopy. Shortly after the first discovery of a transiting exoplanet - HD 209458b by Charbonneau et al. (2000), the first detection of an exoplanet atmosphere was achieved by Charbonneau et al. (2002) with the Hubble Space Telescope (HST). This revealed atomic sodium absorption in its atmosphere. Sing et al. (2008) reanalysed the HST data and obtained an optical transmission spectrum which shows a broad Na I absorption profile. Snellen et al. (2008, hereafter S2008) confirmed the Na I absorption in HD 209458b using transit spectra taken with the High Dispersion Spectrograph on the Subaru telescope - this is one of the first detections of exoplanet atmospheres from the ground. In addition to the atomic sodium, other species are also detected in the HD 209458b atmosphere, such as atomic hydrogen, oxygen and carbon (Vidal-Madjar et al. 2003, 2004).

Apart from HD 209458b, another extensively studied exoplanet is HD 189733b. Redfield et al. (2008) presented the first atmospheric detection for this planet. The detection, made with the High Resolution Spectrograph on the HobbyEberly Telescope, reveals a Na I absorption feature. Pont et al. (2013) showed the full transmission spectrum from the UV to infrared wavelengths and revealed sodium and potassium absorption as well as a strong Rayleigh scattering. Recently, Wyttenbach et al. (2015, hereafter W2015) obtained the spectrally resolved $\mathrm{Na}$ I transmission spectrum of HD 189733b using the High Accuracy Radial velocity Planet Searcher (HARPS) mounted on the ESO-3.6 m telescope (Mayor et al. 2003). These HARPS data are also used by Louden \& Wheatley (2015) for the study of wind and rotation, and by Barnes et al. (2016) for studying excess transit absorption at $\mathrm{H} \alpha$ and $\mathrm{Ca}$ II $\mathrm{H} \& \mathrm{~K}$ line cores.

Transit spectroscopy not only plays an important role in the discovery of atmospheric species, but also has great potential for characterising the physical condition of atmospheres. For example, Snellen et al. (2010) detected a strong wind from the dayside to night-side with a speed of $2 \mathrm{~km} \mathrm{~s}^{-1}$ for HD $209458 \mathrm{~b}$ using the cross-correlation method of the CO lines. Brogi et al. (2016) applied a similar method to HD 189733b and detected a wind speed of $1.7 \mathrm{~km} \mathrm{~s}^{-1}$ using the $\mathrm{CO}$ and $\mathrm{H}_{2} \mathrm{O}$ lines. Louden \& Wheatley (2015) also detected the wind speed of HD 189733 b but with the Na D lines.

The transit spectroscopy technique utilises the stellar flux as the background source which penetrates the planetary atmosphere during transit, thus the non-uniformity of the stellar flux across the stellar disk is important for this technique. For instance, the stellar limb-darkening is a crucial parameter. 
Normally, the broadband differential limb-darkening is well treated in exoplanet studies, however, the stellar lines have a different limb-darkening from the continuum. This behaviour is called the centre-to-limb variation (CLV) effect, meaning that the normalised stellar line shows a variation in the line profile from the centre to limb across the stellar disk. The CLV effect can affect the transit spectroscopy studies which use narrow-band light curve measurements as well as spectrally-resolved measurements including the cross-corelation method.

For the solar Fraunhofer lines, there are prominent variations in both line intensity and profile across the solar disk (Athay et al. 1972). In our previous work (Yan et al. 2015), we observed the Earth transiting the Sun using a lunar eclipse to obtain the transmission spectrum of the Earth's atmosphere. We found that the CLV effect in solar lines results in large residuals in the transmission spectrum obtained and that the CLV effect can lead to the false detection of atmospheric species. For example, the detection of $\mathrm{Na}$ I was claimed in some other lunar eclipse observations (Pallé et al. 2009; Vidal-Madjar et al. 2010; Arnold et al. 2014), but was demonstrated to be the result of the CLV effect rather than of actual planetary absorption.

The importance of the CLV effect has been realised in different studies when investigating transmission spectra. For example, Charbonneau et al. (2002) and Redfield et al. (2008) both considered the CLV effect when measuring the sodium in exoplanet atmospheres and concluded that the CLV effect contribution is much smaller than the observed $\mathrm{Na}$ I absorption for their observations. However, with the continuous improvements in instrumentation, observing strategies and data reduction methods, the CLV effect can no longer be neglected. Its manifestation differs for different planetary systems and for different stellar lines. In some cases, the CLV effect can be very strong, becoming crucial for transmission studies.

Czesla et al. (2015, hereafter C2015) studied the CLV effect and used UVES/VLT data of HD 189733b's transit as an example. However, their data are affected by a flare event and the line core is not presented in their paper. The line core is very important for exoplanet characterisation because the planetary absorption is most prominent there. Here, we present the CLV effect of HD 189733b using another data set from HARPS observations and focus on the CLV at the line core.

Our paper is organised as follows. In Sect. 2, we describe the stellar spectral model for the CLV effect. In Sect. 3, we present the HARPS data of the HD 189733b transit and study how the Na I transmission spectrum is affected by the CLV effect. In Sect. 4, we compare the CLV effects of the two extensively studied exoplanet systems, HD 189733b and HD 209458b, in order to illustrate its manifestation in different exoplanet systems. In Sect. 5, we present the general CLV effect during exoplanet transit and how the CLV effect varies with different planetary and stellar parameters, especially the transit impact parameter and the stellar effective temperature.

\section{The CLV model for exoplanet transit}

The model comprises two parts. Firstly we simulate the synthetic stellar spectrum across the stellar disk, and then integrate the unobscured stellar spectrum to obtain the stellar spectrum visible during transit.

\subsection{Stellar spectrum as a function of limb angle}

The stellar spectrum is modelled using the Spectroscopy Made Easy (SME) tool (Piskunov \& Valenti 2017). We use the
MARCS (Gustafsson et al. 2008) stellar atmosphere model which is included in the distribution of the SME package. The non-local thermodynamic equilibrium (non-LTE) effect can also be included in the SME calculation, and this is done by applying the appropriate departure coefficients. For example, the $\mathrm{Na}$ I departure coefficients are from Lind et al. (2011) and Mashonkina et al. (2008). As presented in Piskunov \& Valenti (2017), the SME non-LTE calculation using the MARCS atmosphere grid reproduces well the line profiles of solar $\mathrm{Na}$ I D lines. We used the line lists from VALD database (Ryabchikova et al. 2015).

The stellar spectra were calculated at 21 limb angles $(\theta$, the angle between the normal to the stellar surface and the line of sight). The corresponding $\mu=\cos (\theta)$ values range from zero to one with a step of 0.05 . When calculating the spectrum at the stellar edge, we use $\mu=0.001$ instead of $\mu=0$ to avoid numerical problems (C2015). According to the simulation, the strong stellar lines are always deeper at the stellar disk centre than at the stellar limb.

\subsection{Synthetic spectrum during transit}

In order to obtain the synthetic spectrum of the integrated stellar disk, we divided the disk into elements with a size of $0.01 R_{\mathrm{S}} \times$ $0.01 R_{\mathrm{s}}$. Each of these elements has a $\mu$ value, and so its spectrum is linearly interpolated from the previously calculated spectra at $21 \mu$ values. The synthetic spectrum during transit is calculated by integrating all the surface elements which are not obscured by the planet.

We synthesised two different kinds of spectra: the complete spectrum and the continuum. In this way, we were able to obtain the normalised spectrum by dividing the complete spectrum by the continuum. The CLV calculation is then performed based on these normalied spectra.

\subsection{Other effects}

In addition to the CLV, there are other effects that change the observed line depth during transit.

(1) Rossiter-McLaughlin effect The CLV model in our work does not include the Rossiter-McLaughlin (RM) effect, but this will only have a very small effect on our calculations. The RM effect means that the observed stellar line profile and radial velocity (RV) change during transit because of the stellar rotation (Rossiter 1924; McLaughlin 1924). For a star like HD 189733 with a rotational velocity of $V \sin I=3.1 \mathrm{~km} \mathrm{~s}^{-1}$ (Triaud et al. 2009), the stellar line shape change due to the RM effect is relatively weak. Besides, when using transit spectroscopy to detect the transmission spectrum, normally the RV differences between the observed stellar spectra are corrected by shifting the spectra to a common velocity before analysis. For the transmission light curve method we employed in the following analysis in Sect. 3, the spectra are integrated over a narrow band and thus the line distortion due to the RM effect has a relatively weak effect on our result. Therefore, we did not include the RM effect in the model.

However, we emphasise here that the RM effect is important for spectrally resolved transmission spectroscopy. For instance, Louden \& Wheatley (2015) showed that the blue-shifted Na absorption of HD 189733b measured by Wyttenbach et al. (2015) is likely due to the uncorrected RM effect. Brogi et al. (2016) also showed that the uncorrected RM effect will produce a $\mathrm{CO}$ signal at $2.3 \mu \mathrm{m}$ dominated by the residual of stellar absorption, 
rather than by the true planet signal. In addition to the RM effect, the CLV effect is also very important for these spectrally resolved studies as it changes the observed stellar line profile.

(2) Planet orbital velocity The planet orbital RV varies during transit, for example, the RV shift of HD 189733b during transit is $\pm 16 \mathrm{~km} \mathrm{~s}^{-1}$. The change of orbital RV causes the shift of the planetary absorption line across the stellar line profile, thus the actual absorbed flux by the planetary atmosphere varies with the orbital RV. For example, when the orbital RV equals zero, the planetary absorption line is mainly centred at the stellar line core where the flux is low, thus the flux absorbed by the planet is relatively small; when the absorption line shifts away from the stellar line core, the flux absorbed by the planet becomes relatively larger. This orbital RV effect is mentioned in Albrecht (2008) and Snellen et al. (2011). More recently, Khalafinejad et al. (2017) observed the light curve of a HD $189733 \mathrm{~b}$ transit due to this orbital RV effect using the UVES/VLT data. Generally, the flux change due to the orbital RV is relatively small and thus it is not included in our CLV model. However, depending on the stellar and planetary absorption line profiles and the chosen passband, the planet orbital RV can introduce additional noise into the observed transmission light curve.

\section{The CLV effect in the HD 189733b data}

HARPS observed HD 189733b transits during several nights. These data have been extensively studied, for example, Wyttenbach et al. (2015), Louden \& Wheatley (2015), Di Gloria et al. (2015), Cegla et al. (2016) and Barnes et al. (2016). Three datasets taken at different nights are used by W2015 and Barnes et al. (2016) for studying the Na I D, H $\alpha$ and $\mathrm{Ca}$ II H\&K lines. In this work, we use two datasets: from Night 1 (2006-09-07) and Night 3 (2007-08-28). The Night 2 (2007-07-19) data are not used because they lack sufficient baseline observations before transit.

The standard data reduction is performed using the HARPS Data Reduction Software. The reduced spectrum is onedimensional, instrument response (blaze) and Baycentric Earth Radial Velocity corrected. The wavelength of the reduced spectrum is in the solar system barycentre rest frame.

For telluric correction, we used a similar method to W2015. The telluric correction mainly corrects the $\mathrm{H}_{2} \mathrm{O}$ lines in the Na I D wavelength region. Firstly, we built a telluric reference spectrum for each night using the linear regression method with the out-of-transit spectra (cf. W2015 and Astudillo-Defru \& Rojo 2013, for more details). Secondly, we fitted the strong $\mathrm{H}_{2} \mathrm{O}$ lines in the obtained telluric reference spectra with the corresponding lines in each observed spectrum, so that a telluric transmission spectrum is obtained from the fit solution. Each observed spectrum was then divided by its corresponding telluric transmission spectrum to remove the telluric lines.

\subsection{Observed transmission light curve of $\mathrm{Na}$ I and the CLV correction}

We chose three bandwidths to calculate the line depth: $0.75 \AA$, $1.5 \AA$ and $3.0 \AA$. These bands were centred at the line cores of each $\mathrm{D}_{1}$ and $\mathrm{D}_{2}$ line. The reference passband used is the same as in W2015, that is, 5874.89 5886.89 $\AA$ for the blue part and $5898.89 \sim 5910.89 \AA$ for the red part (Fig. 1). The relative flux of the Na I lines is calculated using the ratio between the flux inside the band centred at the line core $\left(F_{\text {cen }}\right)$ and the flux inside
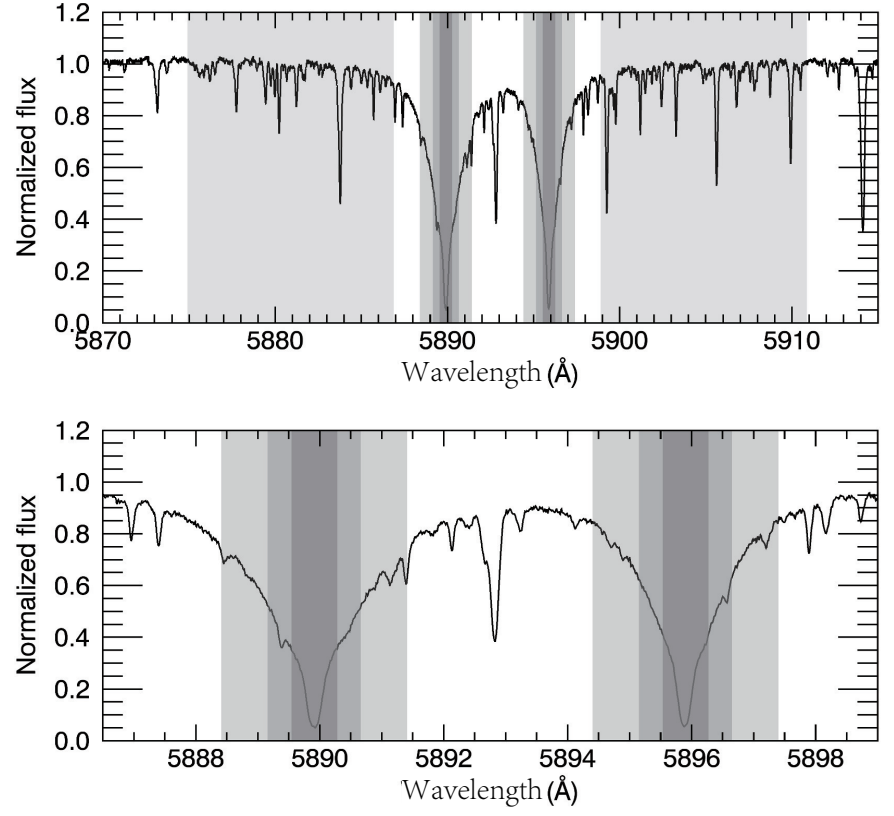

Fig. 1. Observed spectrum of HD 189733. The top panel shows the three bandwidths $(0.75 \AA, 1.5 \AA$ and $3.0 \AA)$ used to measure the relative flux of the NaI D lines. These passbands are centred at the line cores of $D_{1}$ and $D_{2}$. The reference passbands at the blue and red parts of the $\mathrm{Na}$ I D lines are also shown. The bottom panel is the zoom at the the Na I D lines.

the reference bands $\left(F_{\text {red }}\right.$ and $\left.F_{\text {blue }}\right)$ :

$F_{\text {line }}=\frac{2 F_{\text {cen }}}{F_{\text {red }}+F_{\text {blue }}}$.

We normalised the relative flux to unity for all the out-of-transit $F_{\text {line }}$ values. In this way, we can obtain the transmission light curves for $D_{1}$ and $D_{2}$ separately. The light curves of $D_{1}$ and $D_{2}$ are then averaged.

For each night, we obtained one transmission light curve of the Na I D lines. The light curves from Night 1 and Night 3 were then combined and re-ordered according to the phase values. We binned the data points with a 0.002 phase step. In each bin, the data points were weighted by their SNRs. The final observed transmission light curves using different passbands are shown in the first row of Fig. 2 (the red line).

The light curve is the combination of the planetary absorption and the CLV effect. To better characterise the planetary Na I absorption, the CLV effect needs to be corrected. We used the parameters of the HD 189733 system listed in Table 1 as the input to calculate the relative flux change of the Na I lines due to the CLV effect. The modelled light curve of the CLV effect is presented as the black line in the second row of Fig. 2.

We corrected the CLV effect by dividing the observed relative flux light curve with the modelled CLV light curve in order to obtain the actual Na I absorption light curve (see Fig. 2). The $\mathrm{Na}$ I absorption depth is then calculated by fitting with a modelled Na I light curve. The best-fit model of $\mathrm{Na}$ I is plotted as the black line in the third row of Fig. 2.

In Fig. 2, we also plot the combined model (the combination of the CLV model and the best-fit Na I absorption) as the black line in the first row. Additionally, by dividing the observed transmission light curve with the best-fit Na I absorption model, we were able to obtain the observed CLV effect (the red line in the second row of Fig. 2. We emphasise here that the modelled CLV effect light curve is directly from the model and not from the fit 

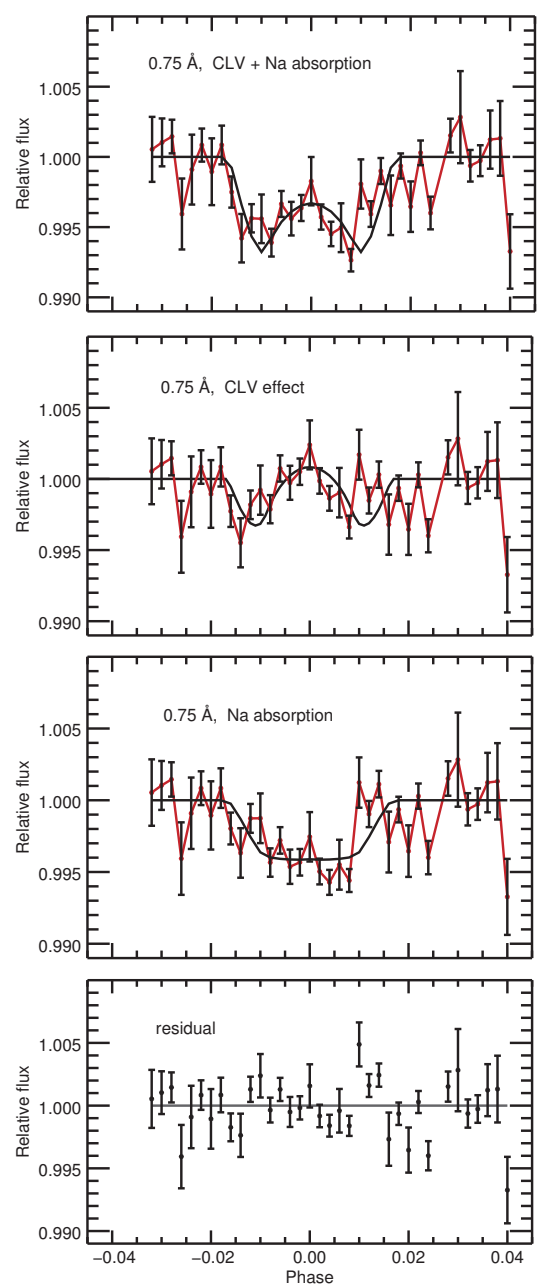
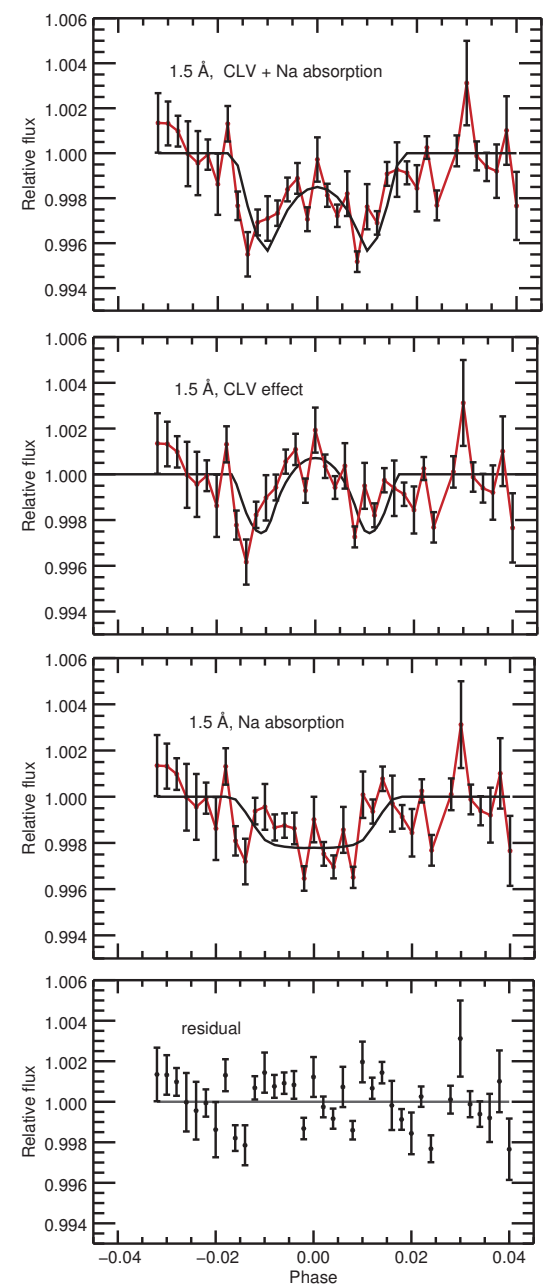
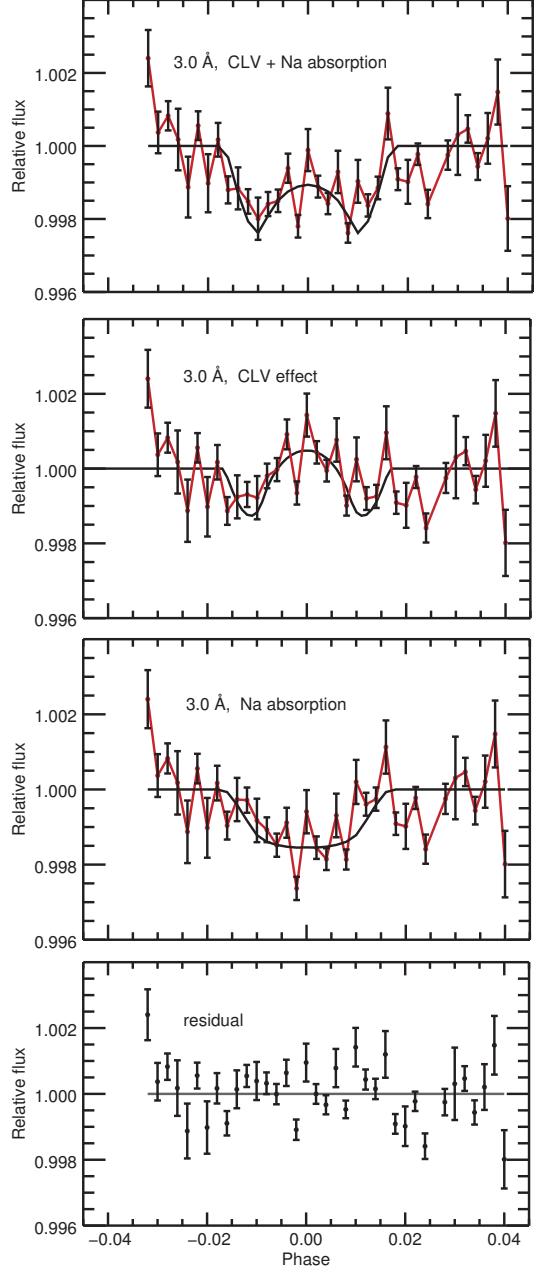

Fig. 2. First row: observed relative flux of the Na I $\mathrm{D}$ lines. This is the final observed transmission light curve from our data reduction. The data are from the HARPS observations of HD 189733b. The data points are the average of $\mathrm{D}_{1}$ and $\mathrm{D}_{2}$ lines and are binned every 0.02 phase step. The black line is the modelled transmission light curve which is the combination of the CLV effect and the best-fit Na I absorption. Second row: CLV light curve. The black line is the modelled CLV effect and the red line is the observed light curve which is obtained by removing the Na I absorption model from the observed transmission light curve. Third row: Na I absorption light curve. The red line is the observed light curve with the modelled CLV effect removed and the black line is the best-fit Na I absorption light curve. Fourth row: residuals between the observed data (the top row) and the model. The three columns correspond to the observed flux measured using the band widths of $0.75 \AA$, $1.5 \AA$ and $3.0 \AA$, respectively. The errorbars represent the photon noise within the passband.

Table 1. Parameters of the HD 189733b system.

\begin{tabular}{lcc}
\hline \hline Parameter & Symbol [unit] & Value \\
\hline The star & & \\
Effective temperature & $T_{\text {eff }}[K]$ & $5040 \pm 50$ \\
Radius & $R_{\mathrm{S}}\left[R_{\odot}\right]$ & $0.756 \pm 0.018$ \\
Mass & $M_{\mathrm{s}}\left[M_{\odot}\right]$ & $0.806 \pm 0.048$ \\
Surface gravity & $\log \left(g\left[\mathrm{~cm} \mathrm{~s}^{-2}\right]\right)$ & $4.587_{-0.015}^{+0.015}$ \\
Metallicity & {$[\mathrm{M} / \mathrm{H}][\mathrm{dex}]$} & $-0.03 \pm 0.08$ \\
& & \\
The planet & & \\
Radius ratio & $R_{\mathrm{p}} / R_{\mathrm{S}}$ & $0.15463 \pm 0.00022$ \\
Orbital semi-major axis & $a\left[R_{\mathrm{s}}\right]$ & $8.81 \pm 0.06$ \\
Orbital inclination & $i$ [degrees] & $85.58 \pm 0.06$ \\
Orbital period & $P$ [days] & 2.21857567 \\
Transit impact parameter & $b\left[R_{\mathrm{s}}\right]$ & $0.680 \pm 0.005$ \\
Transit epoch (BJD) & $T_{0}$ [days] & 2454279.436714 \\
\hline
\end{tabular}

Notes. The transit epoch and the period are from Agol et al. (2010) and all the other values are from Torres et al. (2008). procedure. The fit procedure is only performed for the $\mathrm{Na}$ I absorption light curve. Once the best-fit $\mathrm{Na}$ I absorption model was determined, the combined model and the observed CLV light curve were then obtained directly.

Table 3 presents numeric values of the results. The obtained $\mathrm{Na}$ I absorption depths are $0.414 \pm 0.032 \%, 0.222 \pm 0.018 \%$ and $0.155 \pm 0.011 \%$ at the $0.75 \AA, 1.5 \AA$ and $3.0 \AA$ bands. Here, the CLV effect is presented as three values: mid-transit (phase $=0$ ), limb-transit (where the line depth change is the largest and the planet transits mainly the limb part of the stellar disk, phase $\approx 0.01$ for HD 189733b), overall-transit (average of all the line depth changes in transit). We introduced this overall-transit value because combining all the in transit spectra is a commonly used method for exoplanet atmosphere detection. Here a positive value represents an absorption feature (i.e. the line depth is deeper compared to out of transit), while a negative value represents an excess in line depth compared to out of transit. The last column of the table is the overall CLV contribution (the ratio of the overall-transit values to the actual $\mathrm{Na}$ I absorption depth). 


\subsection{Conclusions for HD $189733 b$}

The observed transmission light curve can be well fitted with the combined model (CLV plus Na I absorption). If we fit the light curve with only the Na I absorption model, then the best-fit absorption depths are $0.512 \%, 0.292 \%$ and $0.175 \%$ at the $0.75 \AA$, $1.5 \AA$ and $3.0 \AA$ bands, respectively. These absorption depths are larger than the absorption depths obtained with the combined model, for example, the depth at the $1.5 \AA$ band is larger by a factor of about one third. The observed light curves fit better with the combined model than with only the $\mathrm{Na}$ I absorption model. For example, the reduced chi-square from the best-fit at the $1.5 \AA$ band is 3.3 for the model without CLV effect while the reduced chi-square is 2.6 for the combined model. Therefore, we conclude that the model with the CLV effect included fits better with the observed light curve and makes the measurement of Na I absorption more precise. For the case of HD 189733b, the $\mathrm{Na}$ I absorption depth is overestimated if the CLV effect was not considered, which will result in a considerable overestimation of the Na I abundance.

Although the CLV value drops with a broader bandwidth, the planetary absorption values also reduce. Thus, the CLV effect compared with the actual planetary absorption does not necessary become less important with an increasing bandwidth. For HD 189733b, the ratios of the overall-transit CLV value to the $\mathrm{Na}$ absorption value are similar for the three band widths (cf. the last column in Table 3). Here one should keep in mind that the CLV effect is wavelength dependent. If this correction is not properly taken into account, the obtained wavelength dependent absorption line profile will deviate from its actual shape, influencing the determination of the atmosphere physical conditions (e.g. temperature-pressure profile).

The above calculations are for the combination of the Na I D doublet. We also provide separate results for $\mathrm{D}_{1}$ and $\mathrm{D}_{2}$ in Fig. 3 . In general, the $D_{1}$ and $D_{2}$ lines have similar Na I absorption depth and CLV feature. Their difference is that both the planetary absorption and the CLV effect for $\mathrm{D}_{2}$ are slightly stronger than for $D_{1}$. For example, for the $0.75 \AA$ band, the CLV effect (overall-transit value) is $0.125 \%$ for $\mathrm{D}_{2}$ while it is $0.115 \%$ for $\mathrm{D}_{1}$, and the planetary absorption depth is $0.470 \%$ for $\mathrm{D}_{2}$ while it is $0.359 \%$ for $\mathrm{D}_{1}$.

\subsubsection{CLV for $M g I b_{1}$ line}

Unlike the $\mathrm{Na}$ I $\mathrm{D}$ resonance lines, there is usually no planetary absorption at $\mathrm{Mg}$ I $\mathrm{b}$ lines. This implies that we can use the $\mathrm{Mg}$ I b line as the compararison line to confirm the CLV effect. Figure 4 is the observed result for the $M g I b_{1}$ line along with the CLV model. The line centre is at $5183.59 \AA$ (in the solar system barycentric rest frame) and the reference bands are $5175.00 \sim 5180.00 \AA$ and $5187.00 \sim 5191.00 \AA$. We did not apply the telluric correction for $\mathrm{Mg} I \mathrm{~b}_{1}$ because the telluric absorption is relatively weak in this wavelength range. This figure shows that the observed light curve of the $\mathrm{Mg}$ I $\mathrm{b}$ line is consistent with the CLV model.

\subsubsection{Comparison with previous results from HARPS observations}

When comparing the results with those in W2015, we find that our Na I absorption depths (without the CLV correction) are larger than theirs. For example, at the $0.75 \AA$ bandwidth, the average depth of Night 1 and Night 3 in W2015 is $0.40 \%$ while

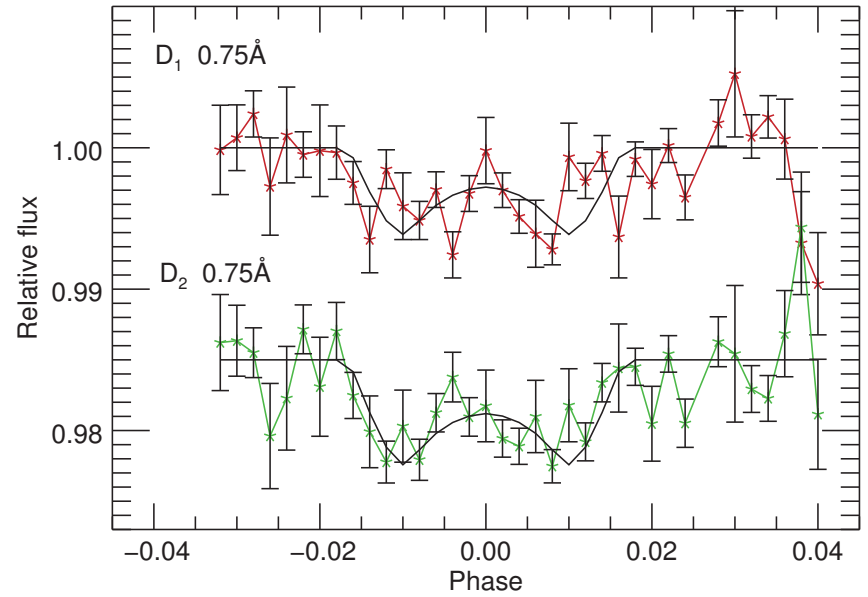

Fig. 3. Observed transmission light curves for $D_{1}$ and $D_{2}$ measured using a $0.75 \AA$ band. The $\mathrm{D}_{2}$ curve is shifted down by 0.15 . The data is from HARPS observations of HD 189733b's transit and are binned every 0.02 phase step. The black solid lines are the best-fit combined models (the CLV effect plus the $\mathrm{Na}$ I absorption). The average of these two light curves is the upper-left plot in Fig. 2.

it is $0.512 \%$ in our work. Barnes et al. (2016) also calculated the $\mathrm{Na}$ I D lines' absorption depth and their result is $0.45 \%$ (average of Night 1 and Night 3) which is also larger than in W2015 but smaller than ours. The differences are probably due to the data reduction method, however, these differences are not significant considering the measurement uncertainties. When fitting the $\mathrm{Na}$ I absorption, we used light curve models which include the limbdarkening within the bandwidths while the other two papers use a box light curve model.

As for the CLV effect, there is no obvious CLV feature in the light curve in W2015 (Fig. 3 of their paper), while the light curve in Barnes et al. (2016, Fig. 6 of their paper) shows a similar CLV feature to that in our work. In general, the light curves in Barnes et al. (2016) and our work agree more closely than with W2015.

\section{Comparison of the CLV effect for HD 189733b and HD 209458b}

The actual CLV effect varies between different exoplanet systems. In order to illustrate and study the different manifestations of the CLV effect, we present the CLV of the HD 209458b system and compare it with the HD 189733 b system. These two transiting exoplanet systems have bright host stars and have been extensively studied, thus they serve as typical samples for the study the CLV effect.

We modelled the CLV effect of HD 209458b using the parameters shown in Table 2. In order to compare the strengths of the CLV effect with the $\mathrm{NaI}$ absorption, we adopted the measured planetary absorption values from (Snellen et al. 2008, S2008). The results are shown in Fig. 5 and Table 4. We note that the calculation in S2008 does not correct for the CLV effect. However, since the overall CLV contribution is relatively small (the value is at the noise level of their observation data), adopting the S2008's value as the Na I absorption is justified in our study.

In order to compare the two systems, we plot their CLV models together with the $\mathrm{Na}$ absorption at three different bandwidths in Fig. 5. There are several sgnificant differences between the CLV effects of the two planetary systems. 

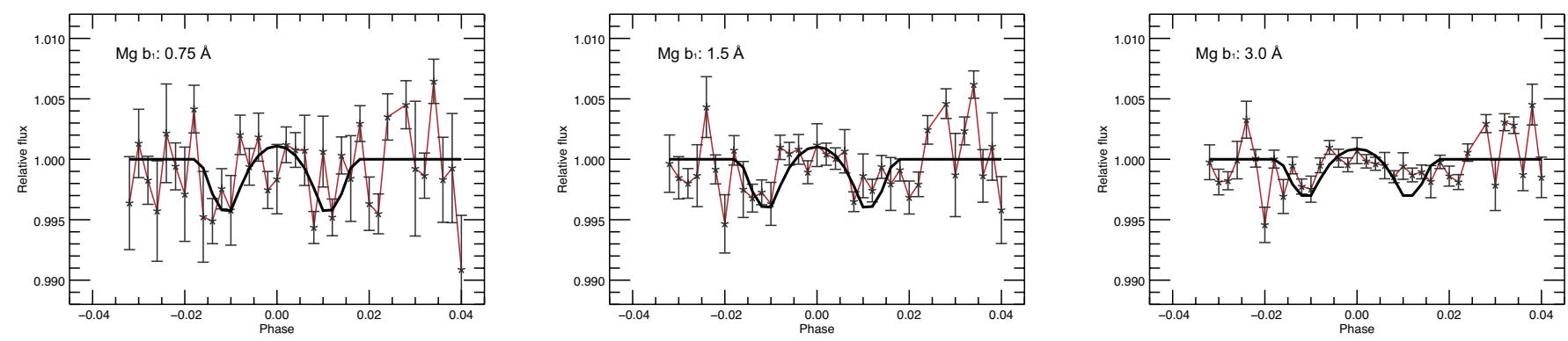

Fig. 4. Observed light curves for the $\mathrm{Mg} \mathrm{I} \mathrm{b}_{1}$ line (red lines). The black line is the CLV model.
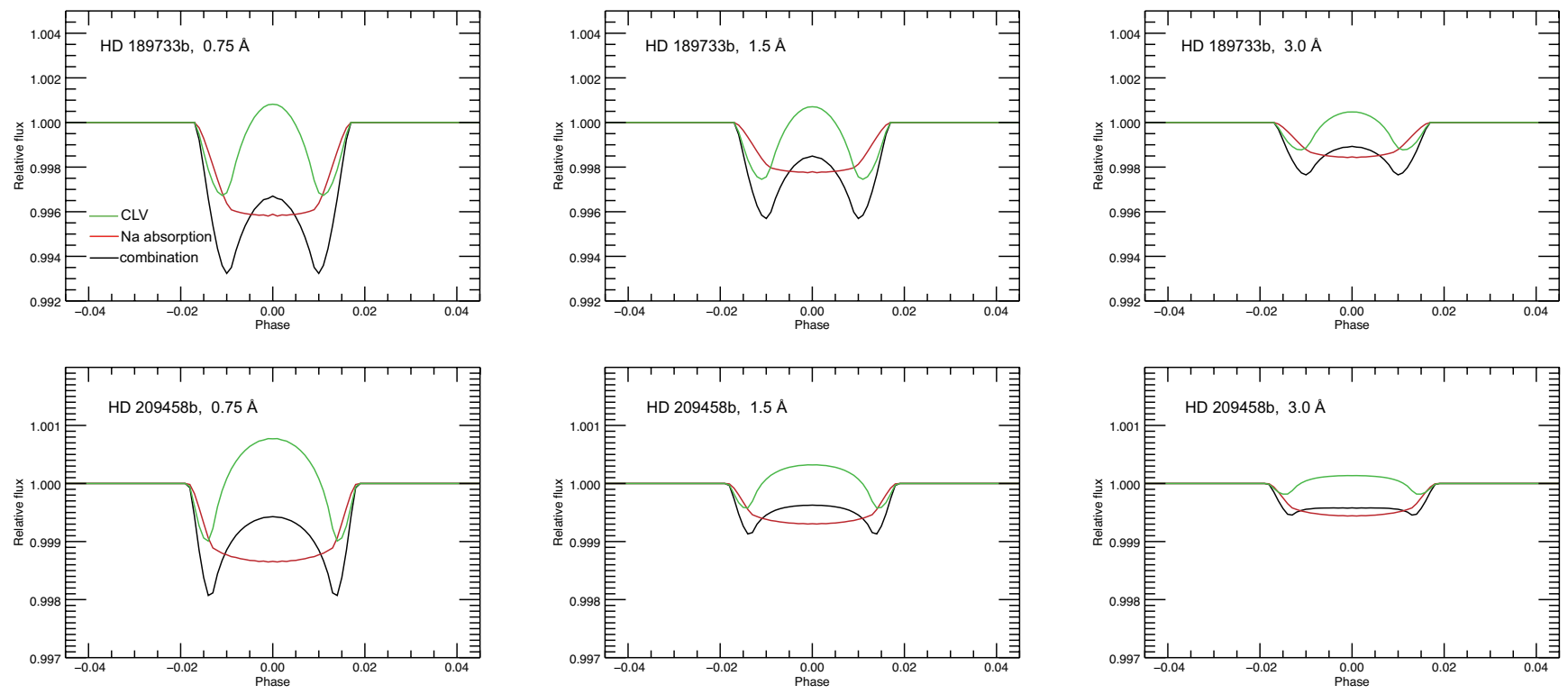

Fig. 5. Comparison of the CLV effect between HD $189733 \mathrm{~b}$ and HD 209458b. Here the calculations are for the Na I D lines (average of $\mathrm{D}_{1}$ and $\mathrm{D}_{2}$ ). The first row is for HD $189733 \mathrm{~b}$ and the second row is for HD 209458b. The three columns are for the bandwidths of $0.75 \AA$, $1.5 \AA$ and $3.0 \AA$. The green lines are the modelled CLV light curves while the red lines are the Na I absorption light curves, and the black lines are the combination of the two. We note that the y axis scales are different for the two rows: the values for HD 189733b are significantly larger than for HD 209458b.

Table 2. Parameters of the HD 209458b system.

\begin{tabular}{lcc}
\hline \hline Parameter & Symbol [unit] & Value \\
\hline The star & $T_{\text {eff }}[\mathrm{K}]$ & $6065 \pm 50$ \\
Effective temperature & $R_{\mathrm{s}}\left[R_{\odot}\right]$ & $1.155_{-0.016}^{+0.014}$ \\
Radius & $M_{\mathrm{s}}\left[M_{\odot}\right]$ & $1.119 \pm 0.033$ \\
Mass & $\log \left(g\left[\mathrm{~cm} \mathrm{~s}^{-2}\right]\right)$ & $4.361_{-0.008}^{+0.007}$ \\
Surface gravity & {$[\mathrm{M} / \mathrm{H}][\mathrm{dex}]$} & $0.00 \pm 0.05$ \\
Metallicity & & \\
& & \\
The planet & $R_{\mathrm{p}} / R_{\mathrm{S}}$ & $0.12086 \pm 0.00010$ \\
Radius ratio & $a\left[R_{\mathrm{S}}\right]$ & $8.76 \pm 0.04$ \\
Orbital semi-major axis & $i[\mathrm{degrees}]$ & $86.71 \pm 0.05$ \\
Orbital inclination & $P[$ days $]$ & 3.52474859 \\
Orbital period & $b\left[R_{\mathrm{s}}\right]$ & $0.507 \pm 0.005$ \\
Transit impact parameter & $T_{0}[$ days $]$ & 2452826.628514 \\
Transit epoch (BJD) & & \\
\hline
\end{tabular}

Notes. The transit epoch and the period are from Knutson et al. (2007) and all the other values are from Torres et al. (2008).

The first difference is in the strengths of the CLV effect. By comparing the CLV values in Tables 3 and 4, and Fig. 5, we conclude that the CLV effect for HD 189733 b is significantly larger than for HD 209458b. The difference is mainly attributed to the effective temperature of the host star (i.e. different spectral types). Generally a star with a lower effective temperature has a stronger CLV effect. The planet-to-star radius ratio also contributes to this difference, however, for these two exoplanet systems, this contribution is small considering that their planetto-star radius ratios are similar ( 0.155 for HD $189733 \mathrm{~b}$ and 0.124 for HD 209458b).

The second difference is their behaviour with different bandwidths. The CLV feature weakens with a broader bandwidth for both systems, however, the drop for HD 209458b is significantly larger than for HD 189733b. For example, the limbtransit value at $3.0 \AA$ is $38 \%$ of the value at $0.75 \AA$ bandwidth for HD 189733b, while for HD 209458b the value is $18 \%$. To better demonstrate this difference, we plot the spectral ratio between limb-transit and out-of-transit for the two systems in Fig. 6. From this figure, we can see that the CLV feature for HD $189733 \mathrm{~b}$ extends over a larger wavelength range than for HD 209458b.

The third difference is the overall-transit value (cf. Table 3 and 4). The overall-transit value is positive for HD 189733b (which means the line depth is deeper than out-of-transit), while the value is negative for HD 209458b (which means the line 
F. Yan et al.: Effect of the stellar absorption line centre-to-limb variation on exoplanet transmission spectrum observations

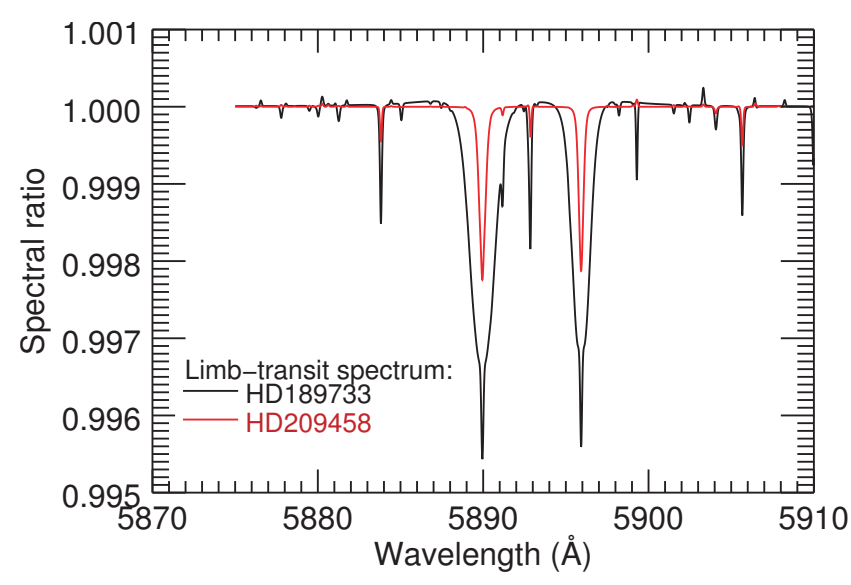

Fig. 6. Ratio of limb-transit spectrum to out-of-transit spectrum. The figure shows clearly that the CLV feature of HD 189733b is stronger and broader than the CLV feature of HD 209458b.

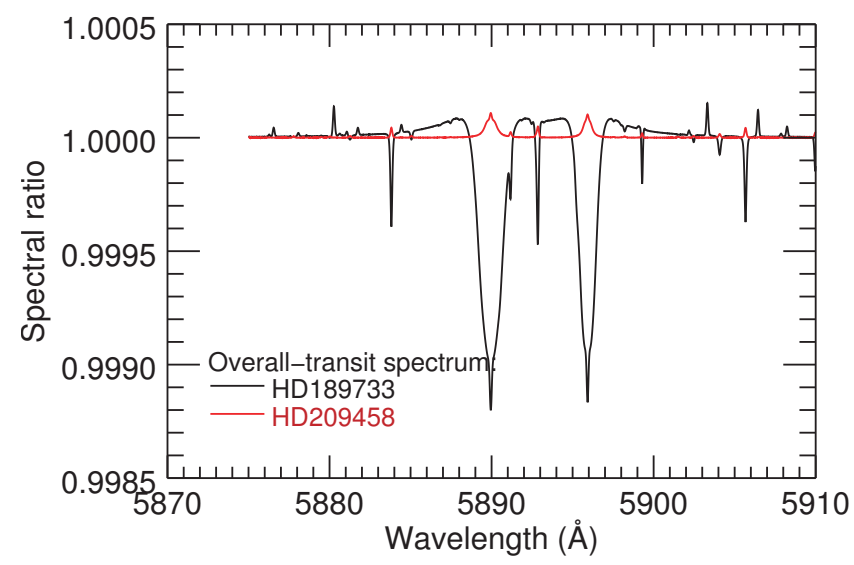

Fig. 7. Ratio of the overall-transit spectrum to the out-of-transit spectrum. Here the overall-transit spectrum is the combination of all the in-transit spectra. This figure shows a significant difference between the overall-transit spectra of HD 189733b and HD 209458b and this difference is mainly attributed to their different transit parameters.

depth is shallower than out-of-transit). Their overall-transit spectra also show significant differences (Fig. 7). The reason is attributed to the transit impact parameter. A larger impact parameter means the planet obscures more limb positions on the stellar disk where the line depth is shallower than out-of-transit and thus the observed line depth is deeper, while a smaller impact parameter means the planet transits more centre positions where the line depth is deeper than out-of-transit and thus the observed line depth is shallower. Since the impact parameter of HD 189733b (0.680) is larger than of HD 209458b (0.507), their overall-transit values are quite different.

\section{General CLV effect during exoplanet transit}

As discussed in Yan et al. (2015), there are a variety of parameters that affect the CLV feature, including stellar parameters and planet transit parameters. In this section, we study two important parameters: the stellar effective temperature and the transit impact parameter.

\subsection{Stellar effective temperature}

The intrinsic variations of stellar lines from the centre to limb are determined by the stellar parameters. Among different stellar

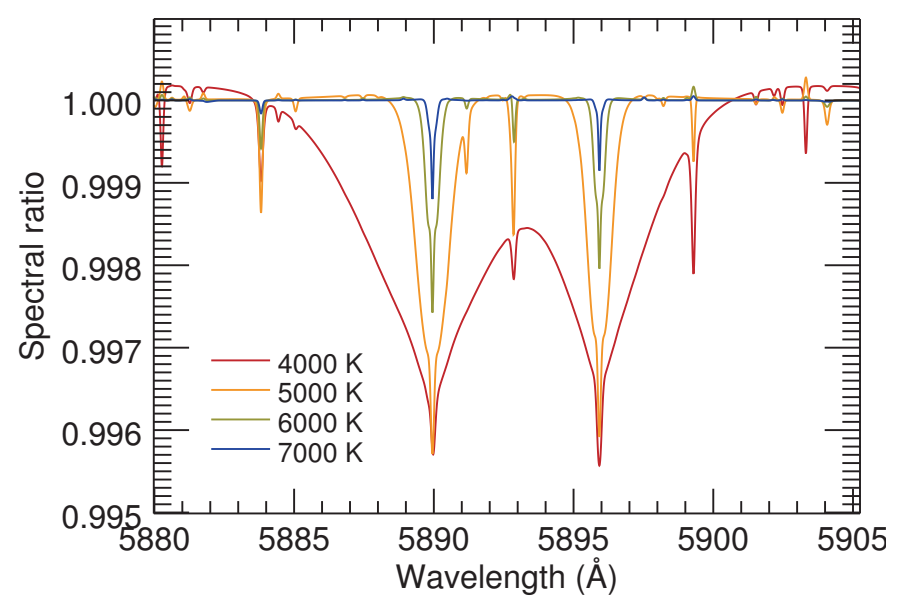

Fig. 8. Ratios of limb-transit spectra to out-of-transit spectra for stars with different $T_{\text {eff }}$. These spectra are simulated using the planetary and stellar parameters described in Sect. 5.1. Here, the limb-transit is defined as the orbital phase with the deepest line depth measured by using a $1.5 \AA$ band centred at the $\mathrm{D}_{2}$ line. This figure presents the change of the CLV effect with $T_{\text {eff }}$.

parameters, the effective temperature has the most significant impact on the CLV feature. The transit light curve of the Na I D line varies significantly with $T_{\text {eff }}$ (cf. Fig. 5 and Table 2 in C2015). To demonstrate the importance of $T_{\text {eff }}$, we constructed the CLV effect models from $4000 \mathrm{~K}$ to $7000 \mathrm{~K}$ with a step of $1000 \mathrm{~K}$. We fixed the following parameters in the CLV model: stellar $\log (g)(4.5)$, stellar metallicity $([\mathrm{M} / \mathrm{H}]=0)$, impact parameter $(b=0)$, planet to star radius ratio $(0.1546$ - same as of HD 189733b). The modelled limb-transit spectra (ratios to the out-of-transit spectra) are plotted in Fig. 8. This figure shows clearly that the CLV effect for the $\mathrm{Na}$ I D lines is stronger for stars with lower $T_{\text {eff }}$.

The stellar effective temperature also affects the manifestation of the CLV effect with different bandwidths. Generally, the CLV value drops with a larger bandwidth, but there are differences between different spectral types. As can been seen in Fig. 8, stars with low effective temperatures have relatively broad CLV features. Thus, for stars with low $T_{\text {eff }}$, the CLV value drops less prominently with increasing bandwidth than for stars with high $T_{\text {eff. }}$.

\subsection{Transit impact parameter}

The planetary parameters are also important for the CLV effect, for example, the planet radius and transit impact parameter. The effect of the planet-to-star radius ratio is straightforward, i.e. a larger planet radius results in a stronger CLV feature. Thus we focus here on the impact parameter $(b)$.

We use the parameters of the HD 189733b system except the $b$ value to model the CLV feature. Figure 9 shows the change of the overall-transit value with different impact parameters. Here, the overall-transit value is the average line depth change for all the in transit spectra measured with a $0.75 \AA$ bandwidth centred at the $\mathrm{NaI} \mathrm{D}_{2}$ line. A positive value means the relative flux is smaller and the line depth is deeper compared to out-oftransit (i.e. similar to a planetary absorption), while a negative value means the relative flux is larger and the line depth is shallower (i.e. opposite to a planetary absorption). Figure 10 shows the transit schematic with different $b$ values and the corresponding CLV light curves. 
Table 3. The measured Na I absorption depth of HD 189733b (CLV effect corrected) and the modelled line depth change due to the CLV effect.

\begin{tabular}{|c|c|c|c|c|c|}
\hline Passband ( & $\begin{array}{l}\text { Planetary absorption depth (\%) } \\
\text { (from this work) }\end{array}$ & mid-transit & $\begin{array}{l}\text { CLV effect model }(\%) \\
\text { limb-transit }\end{array}$ & overall-transit & $\begin{array}{l}\text { CLV overall-transit/ } \\
\text { planetary absorption }\end{array}$ \\
\hline 0.75 & $0.414 \pm 0.032$ & -0.082 & 0.328 & 0.120 & $29 \%$ \\
\hline 1.5 & $0.222 \pm 0.018$ & -0.071 & 0.257 & 0.091 & $41 \%$ \\
\hline 3.0 & $0.155 \pm 0.011$ & -0.048 & 0.126 & 0.038 & $24 \%$ \\
\hline
\end{tabular}

Notes. The values are obtained using the average of $D_{1}$ and $D_{2}$ lines. Here, the CLV effect are presented in three values: mid-transit $($ phase $=0$ ), limb-transit (where the line depth change is the largest) and overall-transit (average of all the line depth changes in transit).

Table 4. Same as Table 3 but for HD 209458b.

\begin{tabular}{|c|c|c|c|c|c|}
\hline Passband $(\AA)$ & $\begin{array}{l}\text { Planetary absorption depth (\%) } \\
\text { (from S2008) }\end{array}$ & mid-transit & $\begin{array}{l}\text { CLV effect model }(\%) \\
\text { limb-transit }\end{array}$ & overall-transit & $\begin{array}{l}\text { CLV overall-transit/ } \\
\text { planetary absorption }\end{array}$ \\
\hline 0.75 & $0.135 \pm 0.017$ & -0.077 & 0.099 & -0.007 & $-5.3 \%$ \\
\hline 1.5 & $0.070 \pm 0.011$ & -0.032 & 0.042 & -0.004 & $-5.7 \%$ \\
\hline 3.0 & $0.056 \pm 0.007$ & -0.014 & 0.018 & -0.002 & $-3.6 \%$ \\
\hline
\end{tabular}

Notes. Here the measured Na I absorption depths are from Snellen et al. (2008).

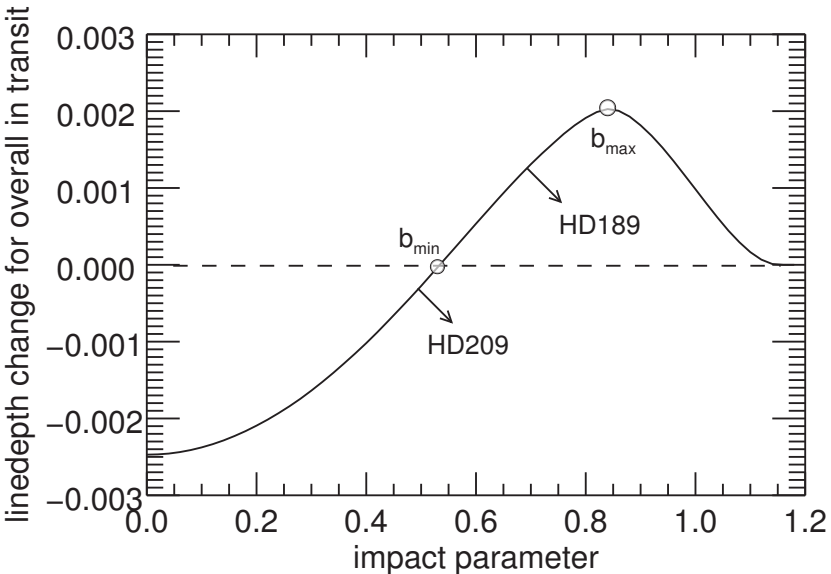

Fig. 9. Overall-transit values at different impact parameters (the "bdiagram"). The values are calculated using a $0.75 \AA$ band centred at the Na I $\mathrm{D}_{2}$ line. The actual b values of HD 189733b and HD 209458b are also indicated.

Below we describe the CLV effect at typical $b$ values:

(1) When $b=0$ (edge-on transit), the overall-transit value is negative and its absolute value is the largest. This means the CLV light curve has the most prominent "bump" feature around mid-transit (the black line in Fig. 10b).

(2) When $b=0.54\left(b_{\min }\right)$, the overall-transit value is zero, which means the average CLV effect during transit does not change the line depth.

(3) The largest overall-transit value appears at $b=0.84\left(b_{\max }\right)$. This positive largest value means the CLV effect makes the line depth much deeper than out-of-transit. The CLV light curve at $b_{\max }$ (the green line in Fig. 10b) is very similar to the planetary absorption light curve. Thus, when the impact parameter of an exoplanet transit is around $b_{\max }$, the CLV effect could mimic the signal of the atmospheric absorption.

(4) When $b=1$, the CLV effect is relatively weak as the transit is grazing and only a small part of the stellar disk is obscured.

We also calculated the $b_{\max }$ and $b_{\min }$ values for other bandwidths $(1.5 \AA$ and $3.0 \AA)$ and the $\mathrm{D}_{1}$ line. The results deviate only slightly (about 0.02 ) from the values of $\mathrm{D}_{2} 0.75 \AA$. So this b-diagram represents the general behaviour of the CLV effect at the $\mathrm{Na}$ I $\mathrm{D}$ doublets.

For stars with different $T_{\text {eff }}$, we modelled their b-diagrams using the same parameters described in Sect. 5.1. The calculated b-diagrams have the same shape as shown in Fig. 9, only that the actual line depth values are more significant with a decreasing $T_{\text {eff }}$. The $b_{\max }$ and $b_{\min }$ values for $4000 \sim 6000 \mathrm{~K}$ are all similar to those of HD 189733b (the deviations are smaller than 0.02). For a star with $T_{\text {eff }}=7000 \mathrm{~K}$, the $b_{\max }$ and $b_{\min }$ are 0.88 and 0.64 (for $\mathrm{D}_{2} 0.75 \AA$ ), which are different from the values of HD $189733 \mathrm{~b}$.

We also modelled the b-diagrams with different planet to star radius ratios $\left(R_{\mathrm{p}} / R_{\mathrm{S}}\right)$. We changed the $R_{\mathrm{p}} / R_{\mathrm{s}}$ from 0.01 to 0.2 and kept all the other parameters the same as the HD $189733 \mathrm{~b}$ system. The $b_{\min }$ value changes by less than 0.02 and the $b_{\max }$ value decreases from 0.90 to 0.82 with the increasing $R_{\mathrm{p}} / R_{\mathrm{s}}$.

With this b-diagram, the overall-transit difference between HD 189733 b and HD 209458 b can be easily understood. The actual impact parameter of HD $189733 \mathrm{~b}$ is 0.680 which is larger than $b_{\min }$ and thus the CLV effect makes the overall-transit line depth deeper. The impact parameter of HD 209458b is 0.507 which is smaller than but close to $b_{\min }$, so the CLV effect results in a shallower line depth and the effect is relatively weak.

\subsection{Stellar atmosphere model}

The modelled CLV feature depends on the stellar atmosphere model. Normally a one-dimensional LTE stellar model can produce a reasonably realastic CLV feature, however, non-LTE and three-dimensional (3D) models can produce more precise results. For the Sun, Allende Prieto et al. (2004) used the CLV of solar lines to test the non-LTE calculations and Koesterke et al. (2008) studied the solar CLV with a 3D model. Recently, Dravins et al. (2016) used the transit spectrum of HD 209458b to study the CLV of Fe I lines with a 3D model.

In our work, we employed a one-dimensional non-LTE model using the SME tool. Compared to the LTE calculation, the non-LTE calculation results in a less prominent CLV effect for the $\mathrm{NaI} \mathrm{D}$ lines. Figure 11 shows the comparison between the LTE and non-LTE cases. The CLV light curves in the figure are calculated for the $\mathrm{D}_{2}$ line $(1.5 \AA$ band) using the parameters 
F. Yan et al.: Effect of the stellar absorption line centre-to-limb variation on exoplanet transmission spectrum observations

(a)
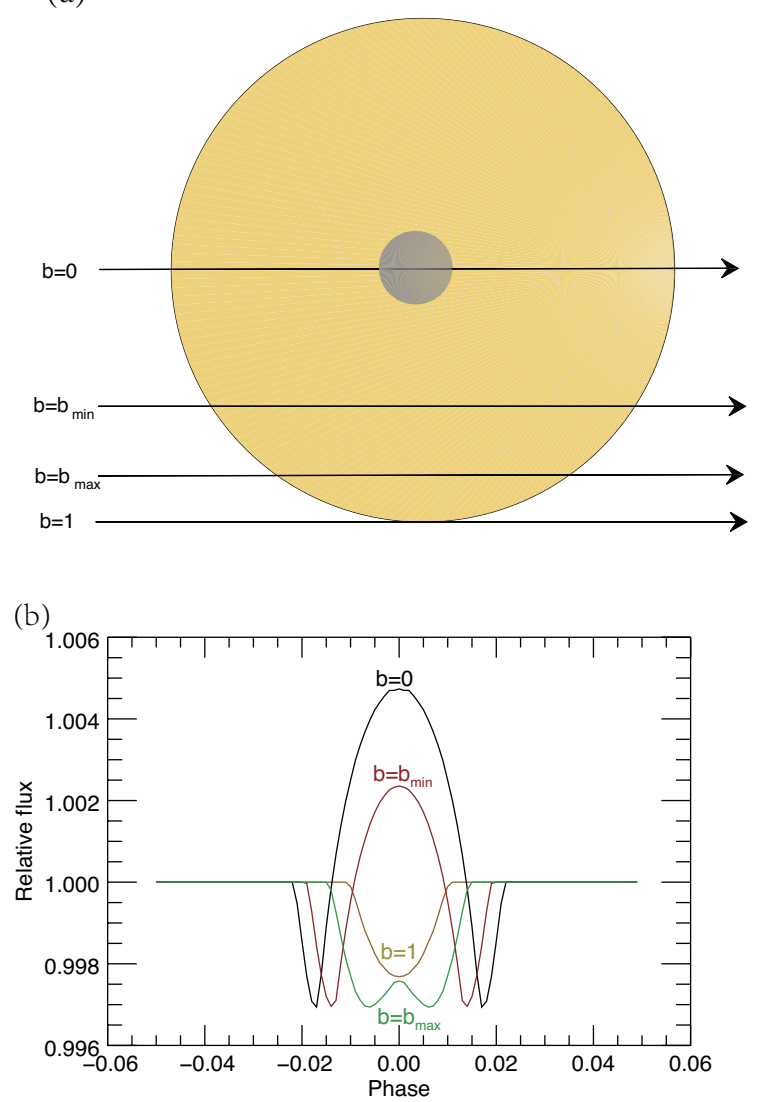

Fig. 10. a) Transit schematic with different impact parameters. b) The

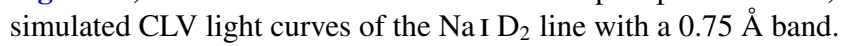

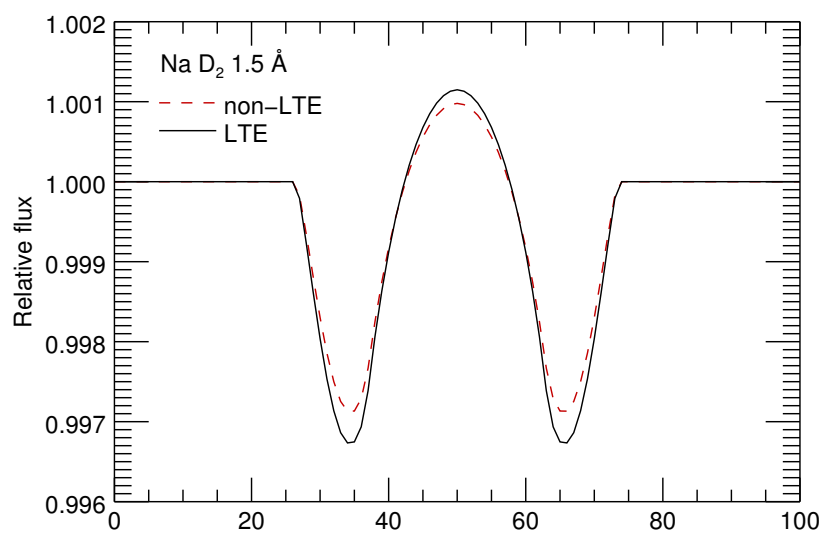

Fig. 11. CLV light curves of $D_{2}$ line from LTE and non-LTE calculations. Here the simulations use the parameters of the HD 189733b system.

of the HD $189733 \mathrm{~b}$ system. The same Na abundance value was used in the LTE and non-LTE calculations.

\subsection{CLV effect for low-mass stars}

In Sect. 5.1, we modelled the CLV effects for stars with $T_{\text {eff }}$ ranging from $4000 \mathrm{~K}$ to $7000 \mathrm{~K}$. However, low-mass stars with lower $T_{\text {eff }}$ are very interesting targets for exoplanet atmosphere studies given their low masses and small radius which make the planet detection and atmospheric characterisation easier. For instance, the recently discovered terrestrial exoplanets around TRAPPIST-1 (Gillon et al. 2016) and Proxima

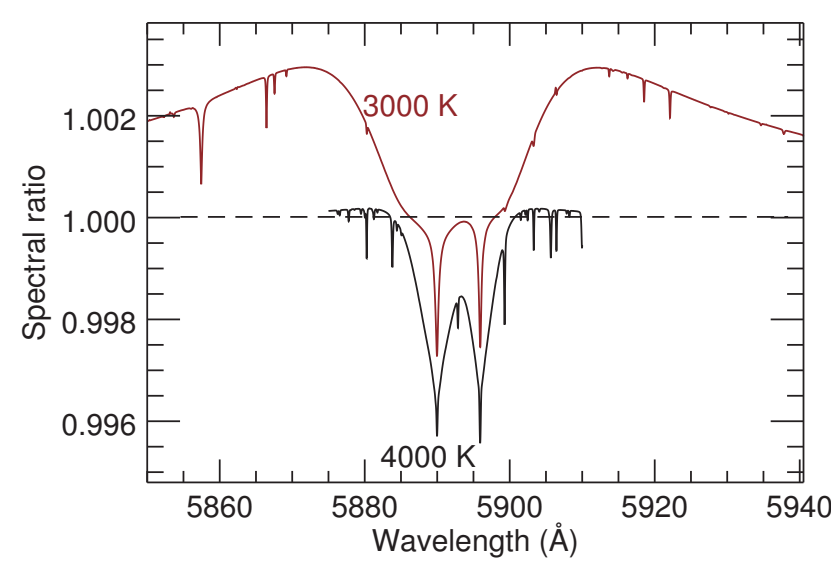

Fig. 12. Spectral ratios of limb-transit to out-of-transit for a low-mass star with $T_{\text {eff }}=3000 \mathrm{~K}$. The spectral ratio for a star with $T_{\text {eff }}=4000 \mathrm{~K}$ is also plotted for comparison. One can see a different behaviour of the line wing for $3000 \mathrm{~K}$. The wavelength range here is larger than in Fig. 8 in order to give a clearer view of the broad line profile.

Centauri (Anglada-Escudé et al. 2016) are both orbiting M-type stars. The CLV effect for low-mass stars is different compared to other spectral types. We synthesize the stellar spectrum for low-mass stars using the SME tool with the MARCS2012 stellar model (which has a $T_{\text {eff }}$ grid from $2500 \mathrm{~K}$ to $8000 \mathrm{~K}$ ) and the VALD line list (which is retrieved with a stellar $T_{\text {eff }}$ of $3500 \mathrm{~K}$ ). The stellar model and line list are valid for low $T_{\mathrm{eff}}$. In Fig. 12, we present the result for a star with $T_{\text {eff }}=3000 \mathrm{~K}$ and $\log (g)=5.2$ (all other parameters are the same as in Sect. 5.1). The limb-transit spectrum for a low-mass star has a different behaviour between line core and line wing. As shown in the figure, at the line wing with wavelength smaller than $5886 \AA$ or larger than $5898 \AA$, the line depth for the limb-transit spectrum is shallower than for out-of-transit. While in the line core regions, the CLV feature is similar to other spectral types. The CLV effect of low-mass stars is particularly important because the $\mathrm{Na}$ I D lines are very broad and deep and have a relatively complicated wavelength-dependent CLV feature.

\section{Conclusions}

We have used the HARPS archive data of HD 189733b transits to show the CLV effect for the Na I D lines. The Na I transmission light curves are the combination of the CLV effect and the actual planetary absorption. After the correction of the CLV effect, the transmission light curve fits the planetary absorption better and the measurement of $\mathrm{NaI}$ absorption becomes more precise. We modelled the CLV effect and the $\mathrm{Na}$ absorption of HD 209458b and HD 189733b and compare the CLV effects of these two benchmark exoplanet systems. Due to the different effective temperatures of their host stars, the CLV effect for HD 189733b is much stronger than for HD 209458b, and the CLV feature of HD 189733b extends to a lager wavelength band than that of HD 209458b. The overall-transit contribution (the average of all the in-transit spectra) of the CLV effect behaves oppositely for the two planetary systems, that is, the overall-transit spectrum compared to the out-of-transit spectrum is deeper for HD 189733b while shallower for HD 209458b. This is attributed to their different transit impact parameters.

We further modelled the CLV effect for planets orbiting stars with various effective temperatures. In general, the Na I D line's CLV effect is stronger and has a broader wavelength range for stars with lower $T_{\text {eff }}$. For low-mass stars with very low $T_{\text {eff }}$, the 
CLV effect are very significant and thus important for transmission spectroscopy. However, spectral modelling for very low $T_{\text {eff }}$ stars is relatively complicated and further work is needed.

The transit impact parameter $(b)$ is important for the CLV effect. The $b$ value determines the trajectory of the planet transit and so the actual CLV effect varies with $b$. We introduced a $b$-diagram which shows the change of the overall-transit value with $b$ (cf. Fig. 9). When $b=0$, the overall-transit value for the Na I D line is negative, which means the stellar line in the overall-transit spectrum is shallower than in the out-of-transit spectrum. At a particular $b$ value $\left(b_{\min }\right)$ where the overall-transit is zero, the net CLV effect has no influence on the stellar line depth. When $b=b_{\max }$ (where the overall-transit value is the largest), the planet transits mostly the limb part of the stellar disk and the line depth is much deeper than in out-of-transit. For an impact parameter close to $b_{\max }$, the CLV effect has a similar light curve to an exoplanet atmosphere absorption. We calculated the $b$-diagrams of $\mathrm{NaI} \mathrm{D}$ lines for stars with $T_{\text {eff }}$ ranging from $4000 \mathrm{~K}$ to $6000 \mathrm{~K}$. According to our simulations, the $b_{\max }$ is generally around 0.84 and $b_{\min }$ is around 0.54 , with deviations smaller than 0.02 .

With the improvement of instrumentation (e.g. future instruments such as ESPRESSO on VLT, HIRES on E-ELT), transit spectroscopy observations will be applied to many exoplanets and the transmission spectra will have a high signal-to-noise ratio. Thus, the CLV effect will become crucial for species detection as well as for the determination of physical conditions using transit spectroscopy.

Future work to improve the CLV model is necessary as the transmission observation becomes more precise. These investigations will benefit from the employment of 3D stellar models, a proper treatment of the non-LTE effects and an improved modelling for stars with very low $T_{\mathrm{eff}}$.

Acknowledgements. We would like to thank Yeisson Osorio, Hans-Günter Ludwig and Karin Lind for helpful discussions of stellar spectral models. We thank the referee for helpful comments. This research is based on data obtained from the ESO Science Archive Facility under request number 217581 and 219943. The observation is made with the HARPS instrument on the ESO 3.6-m telescope at the La Silla Observatory under the programme ID 072.C0488(F) and 079.C-0127(A). This work has made use of the VALD database operated at Uppsala University, the Institute of Astronomy RAS in Moscow, and the University of Vienna.

\section{References}

Agol, E., Cowan, N. B., Knutson, H. A., et al. 2010, ApJ, 721, 1861 Albrecht, S. 2008, Ph.D. Thesis, Leiden Observatory, The Netherlands Allende Prieto, C., Asplund, M., \& Fabiani Bendicho, P. 2004, A\&A, 423, 1109
Anglada-Escudé, G., Amado, P. J., Barnes, J., et al. 2016, Nature, 536, 437 Arnold, L., Ehrenreich, D., Vidal-Madjar, A., et al. 2014, A\&A, 564, A58 Astudillo-Defru, N., \& Rojo, P. 2013, A\&A, 557, A56

Athay, R. G., Lites, B. W., White, O. R., \& Brault, J. W. 1972, Sol. Phys., 24, 18 Barnes, J. R., Haswell, C. A., Staab, D., \& Anglada-Escudé, G. 2016, MNRAS, 462, 1012

Brogi, M., de Kok, R. J., Albrecht, S., et al. 2016, ApJ, 817, 106

Cegla, H. M., Lovis, C., Bourrier, V., et al. 2016, A\&A, 588, A127

Charbonneau, D., Brown, T. M., Latham, D. W., \& Mayor, M. 2000, ApJ, 529, L45

Charbonneau, D., Brown, T. M., Noyes, R. W., \& Gilliland, R. L. 2002, ApJ, 568,377

Czesla, S., Klocová, T., Khalafinejad, S., Wolter, U., \& Schmitt, J. H. M. M. 2015, A\&A, 582, A51

Di Gloria, E., Snellen, I. A. G., \& Albrecht, S. 2015, A\&A, 580, A84

Dravins, D., Ludwig, H.-G., Dahlén, E., \& Pazira, H. 2016, ArXiv e-prints [arXiv: 1607.03489]

Gillon, M., Jehin, E., Lederer, S. M., et al. 2016, Nature, 533, 221

Gustafsson, B., Edvardsson, B., Eriksson, K., et al. 2008, A\&A, 486, 951

Khalafinejad, S., von Essen, C., Hoeijmakers, H. J., et al. 2017, A\&A, 598, A131

Knutson, H. A., Charbonneau, D., Noyes, R. W., Brown, T. M., \& Gilliland, R. L. 2007, ApJ, 655, 564

Koesterke, L., Allende Prieto, C., \& Lambert, D. L. 2008, ApJ, 680, 764

Lind, K., Asplund, M., Barklem, P. S., \& Belyaev, A. K. 2011, A\&A, 528, A103

Louden, T., \& Wheatley, P. J. 2015, ApJ, 814, L24

Mashonkina, L., Zhao, G., Gehren, T., et al. 2008, A\&A, 478, 529

Mayor, M., Pepe, F., Queloz, D., et al. 2003, The Messenger, 114, 20

McLaughlin, D. B. 1924, ApJ, 60

Pallé, E., Zapatero Osorio, M. R., Barrena, R., Montañés-Rodríguez, P., \& Martín, E. L. 2009, Nature, 459, 814

Piskunov, N., \& Valenti, J. A. 2017, A\&A, 597, A16

Pont, F., Sing, D. K., Gibson, N. P., et al. 2013, MNRAS, 432, 2917

Redfield, S., Endl, M., Cochran, W. D., \& Koesterke, L. 2008, ApJ, 673, L87

Rossiter, R. A. 1924, ApJ, 60

Ryabchikova, T., Piskunov, N., Kurucz, R. L., et al. 2015, Phys. Scr., 90, 054005

Sing, D. K., Vidal-Madjar, A., Désert, J.-M., Lecavelier des Etangs, A., \& Ballester, G. 2008, ApJ, 686, 658

Snellen, I. A. G., Albrecht, S., de Mooij, E. J. W., \& Le Poole, R. S. 2008, A\&A, 487,357

Snellen, I. A. G., de Kok, R. J., de Mooij, E. J. W., \& Albrecht, S. 2010, Nature, 465, 1049

Snellen, I., Albrecht, S., de Mooij, E., \& Poole, R. L. 2011, in Molecules in the Atmospheres of Extrasolar Planets, eds. J. P. Beaulieu, S. Dieters, \& G. Tinetti, ASP Conf. Ser. 450, 39

Torres, G., Winn, J. N., \& Holman, M. J. 2008, ApJ, 677, 1324

Triaud, A. H. M. J., Queloz, D., Bouchy, F., et al. 2009, A\&A, 506, 377

Vidal-Madjar, A., Lecavelier des Etangs, A., Désert, J.-M., et al. 2003, Nature, 422, 143

Vidal-Madjar, A., Désert, J.-M., Lecavelier des Etangs, A., et al. 2004, ApJ, 604, L69

Vidal-Madjar, A., Arnold, L., Ehrenreich, D., et al. 2010, A\&A, 523, A57

Wyttenbach, A., Ehrenreich, D., Lovis, C., Udry, S., \& Pepe, F. 2015, A\&A, 577, A62

Yan, F., Fosbury, R. A. E., Petr-Gotzens, M. G., Zhao, G., \& Pallé, E. 2015, A\&A, 574, A94 